\title{
Zusammenhang zwischen dem Küpperschen und Plückerschen Konoid.
}

\author{
Von stud. phil. Paul Ernst in Wien.
}

Als Regelfläche dritter Ordnung mit zwei reellen Kuspidalpunkten läßt sich das durch die Gleichung ${ }^{1}$ )

$$
\left(x^{2}+y^{2}\right)(x-z)+2 r x^{2}=0
$$

dargestellte Küppersche Konoid durch eine reelle Kollineation in in das Plückersche Konoid verwandeln. ${ }^{2}$ )

Ersetzt man in (1) $x$ durch $\xi, y$ durch $\eta_{i}, z$ durch $\zeta+\xi$, so erhält man die Gleichung

$$
\left(\xi^{2}+r^{2}\right) \zeta-2 r \xi^{2}=0,
$$

welche das Plückersche Konoid definiert und durch die Transformation $\xi=\frac{x+y}{\sqrt{2}}, \gamma_{=}=\frac{x-y}{\sqrt{2}}, \zeta=z+r$ in die gewöhnliche $\left.{ }^{3}\right)$ Form

$$
\left(x^{2}+y^{2}\right) z-2 r x y=0
$$

übergeht.

Es geht aber (2) aus (1) auch dadurch hervor, daß man die in $x$ und $y$ quadratischen Glieder von (1) gleich Null setzt, oder geometrisch gedeutet, daf man die ebenen Schnitte $z=k-$ sie sind die Konchoiden von Slus e - durch ihre beiden Tangenten im Doppelpunkt ersetzt.

Daraus folgt der Satz:

Die Tangenten an die Sluseschen Konchoiden auf dem Küppersehen Konoid in den Doppelpunkten der Kurven erfüllen das Plückersche Konoid. \footnotetext{
XVII, S. 309 .

2) Vgl. Zindler, Liniengeometrie II, S. 63 (Sammlung Schubert, LI, Leipzig 1906).

3) Plücker, Neue Geometrie des Raumes, S. 97, Leipzig, Teubner 1868.
}

1) Vgl. meinen Aufsatz „Ửber das Küppersche Konoid“, diese Zeitschrift, 